2 Body positive content on social media aims to challenge mainstream beauty ideals and

3 encourage acceptance and appreciation of all body types. The present study aimed to

4 investigate the effect of viewing body positive Instagram posts on young women's mood and

5 body image. Participants were 195 young women (18-30-years old) who were randomly

6 allocated to view either body positive, thin-ideal, or appearance-neutral Instagram posts.

7 Results showed that brief exposure to body positive posts was associated with improvements

8 in young women's positive mood, body satisfaction and body appreciation, relative to thin-

9 ideal and appearance-neutral posts. Additionally, both thin-ideal and body positive posts were

10 associated with increased self-objectification relative to appearance-neutral posts. Finally,

11 participants showed favourable attitudes towards the body positive accounts with the majority

12 being willing to follow them in the future. It was concluded that body positive content may

13 offer a fruitful avenue for improving young women's body image, although further research

14 is necessary to fully understand the effects on self-objectification. 
\#BoPo on Instagram: An experimental investigation of the effects of viewing body positive content on young women's mood and body image

It is well recognised that the media play a dominant role in influencing perceived social norms and cultural appearance standards, particularly that of the ideal slim female body, commonly referred to as the 'thin-ideal' (Grabe, Ward, \& Hyde, 2008). These appearance ideals have been found to pervade both traditional and social media content (e.g., Conlin \& Bissell, 2014; Tiggemann \& Zaccardo, 2018), and are generally unattainable for most women. A new trend on social media, 'body positivity' (or $\mathrm{BoPo}$ ) aims to challenge these narrow societal prescriptions for female beauty in favour of a broader conceptualisation of beauty, body acceptance of all shapes and sizes, and body appreciation. The current study aimed to investigate the impact of viewing such 'body positive' content on Instagram on women's mood and body image.

\section{Media and Body Image}

According to the Tripartite Influence Model (Thompson, Heinberg, Altabe, \& Tantleff-Dunn, 1999), women internalise the media's unrealistic appearance ideals and engage in appearance comparisons, resulting in dissatisfaction with their own bodies.

Objectification theory (Fredrickson \& Roberts, 1997) offers another framework for understanding the relationship between media images and body image concerns. According to objectification theory, the media's sexual objectification of women socialises women to view their own bodies as objects to be looked at and evaluated based on appearance (known as self-objectification). Both body dissatisfaction and self-objectification have been linked to negative consequences including disordered eating, depression, sexual dysfunction, and substance use (Moradi \& Huang, 2008; Stice \& Shaw, 2002). In support of these theories, a significant literature has shown that exposure to thin-ideal images of women in the media, such as in magazines and on television, can lead to increased thin-ideal internalisation, self- 
objectification, body dissatisfaction, and disordered eating behaviours in women (Grabe et

Newer media sources, such as social media platforms like Facebook and Instagram, can offer a constant stream of carefully curated images and messages promoting the thinideal. Instagram, a photo-based social networking site with 800 million global users who share an average of 95 million photos and videos per day, is most popular amongst 18-29 year old women (Pew Research Center, 2018). A systematic review of the extant literature on social media and body image found that social media use is positively related to body image concerns and disordered eating (Holland \& Tiggemann, 2016). More recent research has shown that it is specifically appearance-focused social media use that is related to body image outcomes, rather than overall time spent on social media (Cohen, Newton-John, \& Slater, 2017, 2018; Meier \& Gray, 2014). For example, correlational studies have shown that engaging in photo-based activities on Facebook (e.g., looking at photos posted by others, sharing one's own photos), following appearance-focused accounts on Instagram, and expending effort and concern in selecting and editing one's selfies before posting them online, are all related to body image concerns in young women (Cohen et al., 2017, 2018; McLean, Paxton, Wertheim, \& Masters, 2015; Meier \& Gray, 2014). Whilst there is less experimental research to date, some experimental studies have shown that exposure to idealised images of women on social media, whether the thin-ideal, fitspiration (lean and

60 toned bodies), or curvy ideals (thin with large breasts and buttocks), led to increased negative 61 mood, body dissatisfaction, and self-objectification in women (Betz \& Ramsey, 2017; Brown

62 \& Tiggemann, 2016; Cohen \& Blaszczynski, 2015; Robinson et al., 2017; Tiggemann \& 63 Zaccardo, 2015). 


\section{Body Positive Social Media}

More recently, there has been a proliferation of 'body positive' content on social media (or ' $B o P o$ ') which aims to challenge the aforementioned narrow appearance ideals and instead represent a diverse array of bodies of different shapes, sizes, colours, features, and abilities, with the presumed aim of fostering body acceptance and appreciation (CwynarHorta, 2016). Unlike traditional media, social media are unique in that their content is usergenerated. This feature allows for bodies that are typically marginalised by society's dominant appearance standards to finally have a voice and be seen. Body positive content has become increasingly popular on social media platforms, particularly on Instagram. A recent search of the hashtag \#bodypositive on Instagram elicited over 6,064,145 posts (Instagram, June 2018). Similar hashtags \#bodypositivity and \#bopo elicited 1,880,753 and 671,063 posts, respectively (Instagram, June 2018). These posts include a variety of quotes, images, and captions, ranging from selfies of women proudly displaying their larger bodies with captions like "it's possible to love your belly rolls, it's possible to have a favourite spot of cellulite", before and after photos of 'real' bodies encouraging awareness of the use of digital alteration in mainstream media, positive quotes like "you are more than a body, go show the world more", and images focusing on body functionality.

This pop-cultural emergence of body positivity on social media coincides with a theoretical shift in the body image literature from a focus on body image disturbance to an exploration of positive body image (Tylka, 2012). Positive body image is a multifaceted construct encompassing a love and respect of the body (Tylka \& Wood-Barcalow, 2015b), and has been operationalised in research as body appreciation (Avalos, Tylka, \& WoodBarcalow, 2005). Preliminary research shows that positive body image may contribute to a host of psychological and physical health benefits. For example, Swami, Weis, Barron, and Furnham (2017) found that positive body image was linked to greater emotional, social, and 
psychological well-being. Similarly, Andrew, Tiggemann, and Clark (2016a, 2016b) found positive body image was positively associated with health-seeking behaviours, intuitive eating and physical activity, and negatively related to dieting, alcohol consumption, and cigarette use. Moreover, there is evidence that body appreciation may play a protective role against the negative impacts of media exposure (Andrew, Tiggemann, \& Clark, 2015; Halliwell, 2013). Accordingly, body appreciation appears to be a fruitful target for interventions that aim to not only reduce women's vulnerability to body dissatisfaction, but also to promote positive body image and its associated positive psychological and physical health benefits (Halliwell, 2015).

Researchers have suggested that in order to improve body appreciation, it is important to provide women with broader conceptualisations of beauty and to encourage women to surround themselves with social networks that foster respect and appreciation for one's own body (Paraskeva, Lewis-Smith, \& Diedrichs, 2017). Accordingly, it is plausible that engaging with body positive content on Instagram, which aims to foster an online community of acceptance and appreciation of all bodies, may be one avenue through which to promote positive body image in young women. A recent study found that women who were exposed to images of full-figured models that did not adhere to the sociocultural thin-ideal reported increases in state body appreciation, compared to those who viewed images of thin models

108 (Williamson \& Karazsia, 2018). Moreover, a recent content analysis of popular body positive 109 accounts on Instagram found that the majority of content analysed depicted a broad range of 110 larger body types, and contained messages that aligned with Tylka and Wood-Barcalow's

111 (2015b) theoretical construct of positive body image (Authors, in preparation). However, to 112 date no research has explicitly investigated the impact of viewing body positive content on 113 Instagram on young women's body image. 
The present study used an experimental design to investigate the effects of exposure

116 to body positive Instagram content on young women's mood, body satisfaction, body

117 appreciation, and self-objectification, in comparison to thin-ideal and appearance-neutral

118 Instagram content. Since body positive content is designed to promote positive body image,

119 and has been shown to align with theoretical definitions of positive body image (Authors, in

120 preparation), we hypothesised that viewing body positive content would result in greater

121 positive mood, body satisfaction, and body appreciation, and reduced self-objectification and

122 negative mood, compared to exposure to thin-ideal content and appearance-neutral content.

123 Finally, given the potential for body positive content to be used as an intervention to improve

124 body image, we were interested in women's attitudes towards these types of accounts, and whether viewing body positive content could have an effect even when controlling for trait

126 levels of body appreciation.

Method

\section{Participants}

Participants were 195 women aged $18-30$ years old $(M=21.69, S D=3.49)$. Just over

130 half of participants (52.8\%) identified as Caucasian, with 34.9\% Asian (including South East

131 Asian), 5.6\% Middle Eastern, 1\% Aboriginal or Torres Strait Islander, 0.5\% African, and

$1325.1 \%$ identifying as 'other' ethnicities. Mean self-reported body mass index (BMI) was 23.08

$133(S D=3.90)$.

134 Procedure

135 Following institutional ethics approval, participants were recruited via fliers and

136 social media pages advertising a study on "Instagram and memory". Upon arrival at the

137 research laboratory, participants were seated in front of a desktop computer and told "We are

138 interested in how your attention and memory are affected when viewing imagery on social 
139 media. After you finish viewing the images you will be asked questions about what you have

140 seen so please pay close attention to the images presented. How you feel can also influence

141 your attention so we are also going to monitor your mood and how you feel throughout the

142 study". After providing informed consent, participants completed measures of pre-exposure

143 state mood and body satisfaction, among distractor items. They were then randomly

144 allocated, via the random allocation function in the Qualtrics survey software, to one of three 145 exposure conditions (body positive, thin-ideal, or appearance-neutral posts). In each

146 condition, participants viewed 20 posts for at least 10 seconds each. Participants then

147 completed post-exposure measures of state self-objectification, state mood and body

148 satisfaction, and state body appreciation among distractor items and memory questions to

149 bolster the cover story. Participants then completed a measure of trait body appreciation,

150 followed by attitudes towards body positive content. Participants were also asked to report

151 their age, ethnicity, and height and weight (used to calculate BMI). Testing sessions lasted

152 approximately 15-20 minutes, and participants received a coffee voucher for their

153 participation. All participants were debriefed on completion of the study.

\section{Measures and Materials}

Experimental manipulation: Post type. Three sets of visual stimuli were used in the study (body positive, thin-ideal, and appearance neutral), each containing four individual

157 Instagram accounts with five posts each (20 posts in total per condition). All posts were

158 sourced from public Instagram accounts. The thin-ideal and body positive posts were selected

159 from an initial pool of 50 body positive and 50 thin-ideal posts (five Instagram accounts per

160 condition with 10 posts each) to provide a reasonable coverage of currently disseminated

161 posts in the designated categories. A pilot study was conducted with 13 independent female

162 raters from the target age group $(M=22.45$ years $S D=2.46)$. Raters were provided with a

163 definition of 'body positive' ['body positive' refers to rejecting unrealistic body ideals and 

Instagram posts tend to depict women proudly posting their unique bodies and quotes about bodyacceptance(e.g., @bodyposipanda, @Ashleygraham, @effyourbeautystandards etc.)], and 'thin-ideal' [ 'idealised images' refer to images of attractive women with thin and toned bodies. Instagram posts of idealised women tend to depict thin women either posing in bikinis, form-fitting or revealing fashion or in fitness attire (e.g., @victoriasecretangels, @kendalljenner, @ gigihadid etc.)], and asked to rate the extent to which each image was representative of its designated category using a visual analogue scale (VAS; $0=$ not at all, $100=$ to a great extent $)$. The accounts and posts rated to be most representative of the conditions were selected for the study (body positive $M=72.31, S D=11.86$; thin-ideal $M=$ 79.77, $S D=10.08)$.

The final thin-ideal stimuli consisted of posts from four popular accounts that were perceived as subscribing to the thin-ideal, and included full body shots of women with thin physiques either posing in bikinis, form-fitting fashion, or fitness attire, as these are typical posts found on Instagram accounts that depict the thin-ideal. The final body positive stimuli consisted of posts from four popular body positive accounts: 1) @ bodyposipanda: images of a larger woman displaying her body with captions about body acceptance, 2) @ omgkenzieee: side by side images of a 'real' woman challenging societal beauty ideals, 3) @ beautyredefined: body positive quotes, and 4) @ nolatrees: images of a 'fat' woman

183 practicing yoga with captions focusing on appreciating what her body can do. This cross

184 section of accounts was selected to represent the different types of posts typically found on body positive accounts. Specifically, 15 of the 20 body positive posts contained women in bikinis, form-fitting fashion, or fitness attire (matching the 20 thin-ideal images except for

187 body type), and five of the images consisted of quotes. The women in the thin-ideal and body 188 positive posts were of similar age to the participants. The appearance-neutral posts consisted 
189 of nature photography typical of Instagram such as plants, marine life, skyscapes, and

190 animals, with no human bodies present. All posts were presented with Instagram borders,

191 names, and captions to enhance ecological validity. However, comments and likes were

192 removed to avoid any confounding effects. Stimuli were presented to participants on a

193 desktop computer screen in a randomised account order with each post displayed for a

194 minimum of 10 seconds before giving participants the option to move to the next image. All

195 images were counterbalanced to control for order effects.

196 State Mood and Body Satisfaction. Computer based visual analogue scales (VAS)

197 were used to measure state mood and body satisfaction both before and immediately after

198 viewing the experimental stimuli. Participants were asked to rate how they feel "right now"

199 by moving a vertical marker to the appropriate point on each horizontal line with end points

200 labelled 'not at all' (0) and 'very much' (100). Participants were asked to rate a series of

201 mood dimensions: depressed, anxious, confident, and happy. Research has found that in low

202 stress situations, positive and negative mood are experienced independently, and therefore

203 should be measured as separate dimensions (Reich, Zautra, \& Davis, 2003). Accordingly,

204 ratings of 'happy' and 'confident' were combined to form a measure of state positive mood,

205 and 'depressed' and 'anxious' combined to form a measure of state negative mood.

206 The body satisfaction dimensions included 'satisfied with my weight', 'satisfied with

207 my overall appearance', and 'satisfied with my body shape', which were combined to form a

208 measure of state body satisfaction. To further disguise the true purpose of the study,

209 participants were also asked about their satisfaction with their romantic relationship, financial

210 status, housing situation, occupation/study, and social life. Previous research has shown VAS

211 to be reliable and sensitive measures of changes in mood and body satisfaction among

212 college women, and thus are ideal for pre-post-experimental designs (Fardouly, Diedrichs,

213 Vartanian, \& Halliwell, 2015; Heinberg \& Thompson, 1995; Prichard \& Tiggemann, 2012). 
214 In the current study, the positive mood scale demonstrated acceptable internal consistency at

215 pre- $(\alpha=.69)$, and post-exposure $(\alpha=.75)$, the negative mood scale demonstrated good

216 internal consistency at pre- $(\alpha=.77)$, and post-exposure $(\alpha=.80)$, and the body satisfaction

217 scale demonstrated good to excellent internal consistency at pre- $(\alpha=.84)$, and post-exposure $218(\alpha=.92)$.

219 State Self-Objectification. A modified version of the Twenty Statements Test

220 (Fredrickson, Roberts, Noll, Quinn, \& Twenge, 1998) was used to measure state selfobjectification. Participants were asked to describe themselves by completing 10 sentences beginning with 'I am'. This implicit measure of state self-objectification has been successfully used in prior experimental research (Calogero, 2013; Harper \& Tiggemann, 2008; Tiggemann \& Boundy, 2008). As per Harper and Tiggemann (2008), two independent researchers who were blind to the hypotheses and experimental conditions coded the responses into one of six categories: 1) body shape and size (e.g., "I am overweight"), 2) other physical appearance (e.g., "I am blonde”), 3) physical competence (e.g, "I am strong”), 4) traits or abilities (e.g., "I am friendly"), 5) states or emotions (e.g., "I am tired”), and 6) miscellaneous or uncodable. State self-objectification was operationalised as the number of responses that fit into the first two categories. This produced a score ranging from 0 to 10 , with higher scores indicating higher levels of self-objectification. There was substantial interrater agreement for appearance items in the first two categories (Cohen's $\kappa=0.75)$. The authors resolved the remaining discrepancies through discussion until consensus was reached. State Body Appreciation. A modified version of the State Body Appreciation Scale2 (SBAS-2; Homan, 2016) was used to assess state body appreciation. The scale was presented as a VAS, requiring participants to rate how they feel "right now" by moving a vertical marker to the appropriate point on each horizontal line with end points labelled 'not at all' (0) and 'very much' (100). The four items include "At this moment, I feel good about 
my body", "At this moment, I feel love for my body", "Right now, I am comfortable in my body", and "Right now, I appreciate the different and unique characteristics of my body".

241 Scores were averaged, with higher scores indicating higher levels of state body appreciation.

242 Homan (2016) examined the factor structure and psychometric properties of the SBAS-2, and

243 found it to be a valid, reliable, and sensitive measure of state body appreciation. For this 244 study the scale showed excellent reliability $(\alpha=.94)$.

Trait Body Appreciation. The Body Appreciation Scale-2 (Tylka \& Wood-

Barcalow, 2015a) was used to measure trait body appreciation. Participants are asked to respond to 10 items on a 5-point scale ranging from 'never' (1) to 'always' (5). Example items include "I respect my body" and "I appreciate the different and unique characteristics of my body". Scores were averaged, with higher scores indicating a higher level of body appreciation. Tylka and Wood-Barcalow (2015a) reported good internal consistency, testretest reliability, and construct validity with a sample of college women. For this study the scale showed excellent reliability $(\alpha=.94)$.

Attitudes towards Body Positive Accounts. All participants were given a definition of body positive accounts and asked how often they currently view body positive content on social media in their everyday lives on a 5-point scale ranging from 'never' (1) to 'always' (5), and how likely they would be to follow such accounts in the future 'very unlikely' (1) to 'very likely' (5). Finally, to ascertain attitudes towards the body positive accounts compared to the thin-ideal accounts, participants in both conditions were presented with an image from each of the four Instagram accounts that they had viewed in their condition and asked to respond to three statements 1) "I like the person who this account belongs to", 2) "I would want to be friends with this person", and 3) "I would want to follow this account" on a 5point scale ranging from 'strongly disagree' (1) to 'strongly agree' (5). Scores were averaged, with higher scores indicating more positive attitudes towards the Instagram accounts they 
264 viewed. For this study the scale showed good reliability (body positive accounts: $\alpha=.89$; thin265 ideal accounts $\alpha=.83$ ).

\section{Results}

\section{Preliminary Analyses}

Available item analysis was used to handle missing data ( $<1 \%$ across all variables). A series of one-way ANOVAs were conducted to ensure that there were no initial differences across the three experimental conditions. There were no significant group differences in age, $F(2,192)=0.47, p=.63$, partial $\eta^{2}=.01$, racial background, $F(2,192)=0.84, p=.43$, partial $\eta^{2}=.01$, BMI, $F(2,191)=0.76, p=.47$, partial $\eta^{2}=.01$, pre-exposure positive mood, $F(2,191)=3.02, p=.05$, partial $\eta^{2}=.03$, pre-exposure negative mood, $F(2,192)=0.01, p>$ .99 , partial $\eta^{2}<.01$, and pre-exposure body satisfaction, $F(2,190)=0.22, p=.80$, partial $\eta^{2}<$ .01. Nor did the conditions differ on trait body appreciation, $F(2,192)=0.14, p=.87$, partial $\eta^{2}<.01$ indicating that this measure had not been reactive to the experimental manipulation. Participants assigned to each condition did not significantly differ in their frequency of viewing body positive posts on social media in their everyday lives $F(2,192)=1.88, p=.16$, partial $\eta^{2}=.02$

\section{State Positive Mood}

The means and standard deviations for each outcome measure per condition are presented in Table 1. A two-way mixed ANOVA was conducted to determine whether changes in positive mood over time were different for those exposed to different types of Instagram posts. There was a statistically significant interaction between type of Instagram exposure and time on positive mood, $F(2,191)=12.34, p<.001$, partial $\eta^{2}=.11$. As seen in increased from pre- to post-exposure for those exposed to body positive posts, $F(1,64)=$ 
$289 \eta^{2}=.14$, whereas for those exposed to thin-ideal Instagram posts, positive mood significantly

290 decreased from pre- to post-exposure, $F(1,64)=9.82, p=.003$, partial $\eta^{2}=.13$.

\section{State Negative Mood}

A two-way mixed ANOVA was conducted to determine whether changes in negative mood over time were different for those exposed to different types of Instagram posts. There was a statistically significant interaction between type of Instagram exposure and time on negative mood, $F(2,192)=3.37, p=.04$, partial $\eta^{2}=.03$. Changes in negative mood over time were significantly different for the different types of exposure, with negative mood increasing following exposure to thin-ideal posts, and decreasing following exposure to both body positive and appearance-neutral posts (see Figure 2). However, simple main effects for each condition were not significant $(p s>.05)$.

State Body Satisfaction

A two-way mixed ANOVA was conducted to determine whether changes in body satisfaction over time were different for those exposed to different types of Instagram posts. There was a statistically significant interaction between type of Instagram exposure and time on body satisfaction, $F(2,190)=31.59, p<.001$, partial $\eta^{2}=.25$. As seen in Figure 3, simple main effect analysis showed that for those exposed to body positive posts, body satisfaction significantly improved from pre- to post-exposure, $F(1,64)=32.32, p<.001$, partial $\eta^{2}=$ .34 , whereas for those exposed to thin-ideal Instagram posts, body satisfaction significantly decreased from pre- to post-exposure, $F(1,64)=25.74, p<.001$, partial $\eta^{2}=.29$. There were no significant differences between pre- and post-exposure body satisfaction for those exposed to appearance-neutral posts $F(1,62)=3.60, p=.06$, partial $\eta^{2}=.06$.

\section{State Body Appreciation} were different following exposure to the different types of Instagram posts. Body 
appreciation scores were significantly different following the different types of exposure $F(2,192)=3.26, p=.04$, partial $\eta^{2}=.03$. As seen in Figure 4, body appreciation scores were highest for those exposed to body positive posts, followed by appearance-neutral posts, with

317 the lowest levels of body appreciation following exposure to thin-ideal posts. Tukey post hoc analysis revealed that body appreciation levels were significantly higher for those exposed to body positive posts compared to thin-ideal posts $(M D=10.72, S E=4.21, p=.03)$, but no other group differences were statistically significant $(p s>.05)$.

\section{State Self-objectification}

A one-way ANOVA was conducted to determine if state self-objectification scores differed across the three exposure conditions. State self-objectification scores were significantly different between the different exposure conditions, $F(2,192)=7.40, p=.001$, partial $\eta^{2}=.07$. As seen in Figure 5, state self-objectification scores were highest for those exposed to body positive posts, followed by the thin-ideal condition, and lowest in the appearance-neutral condition. Tukey post hoc analysis revealed that state self-objectification was significantly higher in the thin-ideal and body positive conditions compared to the appearance-neutral condition $(M D=0.49, S E=0.16, p=.01$; and $M D=0.55, S E=0.14, p<$ .001 respectively). There were no significant differences in state self-objectification scores between those exposed to thin-ideal and body positive posts $(M D=0.06, S E=0.16, p=.92)$. In accordance with previous research (Aubrey, Henson, Hopper, \& Smith, 2009), the valence of each appearance-based statement was further coded as negative (-1; e.g., "I am dumpy"), positive (+1; e.g., "I am cute"), or neutral (0; e.g., "I am brunette"). A one-way ANOVA was conducted to determine if the valence of appearance-related statements differed between the body positive and thin-ideal conditions. Results showed that women who viewed body positive posts made significantly more positive statements about their appearance $(M=$ 
$3380.37, S D=0.84)$ than the women who viewed thin-ideal posts $(M=0.00, S D=$

$3390.79), F(2,192)=5.40, p=.005$, partial $\eta^{2}=.05$.

\section{Controlling for Trait Body Appreciation}

We were interested to see if the effects of viewing body positive versus thin-ideal

342 Instagram posts on state positive and negative mood, state body satisfaction, state body appreciation, and state self-objectification differed when controlling for trait body appreciation. Even when controlling for trait body appreciation, there was a statistically significant interaction between type of Instagram exposure and time on positive mood $F(2$, $190)=12.64, p<.001$, partial $\eta^{2}=.12$, negative mood, $F(2,191)=3.42, p=.04$, partial $\eta^{2}=$ .04 , and body satisfaction, $F(2,189)=31.85, p<.001$, partial $\eta^{2}=.25$. Similarly, ANCOVAs showed that, even after adjustment for trait body appreciation, post-exposure state body appreciation levels were significantly higher following exposure to body positive posts compared to thin-ideal posts, $F(2,191)=6.66, p=.002$, partial $\eta^{2}=.07$, and post-exposure state self-objectification was significantly higher in the thin-ideal and body positive conditions compared to the appearance-neutral condition $F(2,191)=7.54, p=.001$, partial $\eta^{2}=.07$

Attitudes towards Body Positive Accounts

An independent samples $t$ test established that those who viewed body positive accounts formed more positive attitudes towards the women in the accounts they viewed $(M$ $=3.55, S D=0.75)$ compared to those who viewed the thin-ideal posts $(M=2.73, S D=0.75)$, $t(127)=6.17, p<.001$. Moreover, just over half of all participants $(51 \%, n=99)$ said that they were somewhat or very likely to follow body positive accounts in the future, and this

360 likelihood to follow body positive accounts in the future did not differ across conditions

361 (body positive: $M=3.18, S D=1.25$, thin-ideal: $M=3.18, S D=1.21$, appearance-neutral: $M$ $362=3.29, S D=1.32), F(2,192)=0.16, p=.85$. 
The present study aimed to examine the impact of exposure to body positive

Instagram posts on women's state mood, body satisfaction, body appreciation, and self-

objectification relative to thin-ideal and appearance-neutral Instagram posts. In support of the

hypotheses, brief exposure to body positive content on Instagram was associated with improvements in young women's positive mood and body satisfaction, whereas viewing thinideal posts was associated with decreases in positive mood and body satisfaction. Women who viewed body positive content also reported greater body appreciation than women who viewed thin-ideal content. Exposure to appearance-neutral posts had no impact on body image outcomes as expected, but was associated with improvements in positive mood.

373 Although not predicted, this finding was not surprising given that exposure to nature has been found to improve mood (Velarde, Fry, \& Tveit, 2007). Additionally, exposure to both body positive and thin-ideal content was associated with increased state self-objectification relative to exposure to appearance-neutral content.

These findings contribute to the existing research in two important ways. Firstly, they lend experimental support to the growing, yet mostly correlational, body of research on the harmful effects of viewing thin-ideal social media content on women's mood and body image (Holland \& Tiggemann, 2016), providing further support for the application of the Tripartite Influence Model and objectification theory to the social media environment. Secondly, to the

382 best of our knowledge, the present study is the first experimental study to demonstrate that

383 viewing 'body positive' content on Instagram (or BoPo) may improve positive mood, body satisfaction, and body appreciation. In line with the theoretical construct of positive body image, by providing women with broader conceptualisations of beauty and fostering body appreciation, body positive content may offer a practical and cost-effective way to both reduce women's vulnerability to body dissatisfaction, as well as promote positive body image 
388 (Halliwell, 2015; Paraskeva et al., 2017). The fact that these results held even when controlling for trait body appreciation indicates that brief exposure to body positive content can have an immediate positive impact on a woman's body image regardless of her trait

391 levels of body appreciation.

This study also examined the effects of viewing body positive content on young

393

394

395

396

397

398

399

400

401

402

403

404

405

406

407

408

409

410

411

412 women's state self-objectification. Interestingly, women reported more appearance-related statements after viewing both thin-ideal and body positive posts compared to the appearanceneutral posts, and there were no differences between the thin-ideal and body positive conditions. Previous correlational research have found that recalled experiences of both appearance criticisms and compliments were associated with higher levels of selfobjectification (Calogero, Herbozo, \& Thompson, 2009; Slater \& Tiggemann, 2015). Although these studies were investigating the effects of appearance commentary made by others, and not self-referential comments, the findings converge with the results of the present study to suggest that any focus on one's appearance, whether positive or negative, may be associated with greater state self-objectification. This finding is also understandable given that body positive content also exists on the photo-based platform of Instagram and contains images of women's bodies in revealing clothing (Authors, in preparation), as well as captions that make explicit references to aspects of appearance like 'cellulite', 'belly rolls', 'curvy', and 'fat'. Research shows that viewing objectifying images and objectifying words can separately prime state self-objectification (Harper \& Tiggemann, 2008; Roberts \& Gettman, 2004), and therefore, despite its positive intentions, it is possible that viewing body positive content may be associated with higher state self-objectification in young women just like other forms of appearance-focused social media (Betz \& Ramsey, 2017; Cohen et al., 2017). Given the potential ramifications of self-objectification on body shame, depression and eating disorder symptomatology (Moradi \& Huang, 2008), future longitudinal research is 
413 needed to understand the long-term effects of following body positive content on Instagram,

414 in terms of body image outcomes, self-objectification, and general well-being.

415 Notably, when the appearance-related statements were re-analysed in terms of valence

416 (Aubrey et al., 2009; Harrison \& Fredrickson, 2003), we found that the women who viewed

417 body positive posts made more positive statements about their appearance than the women

418 who viewed thin-ideal posts. Whilst self-objectification is typically related to negative body

419 image (Halliwell, 2015), it is possible for a women to self-objectify and be happy with her

420 appearance (Aubrey et al., 2009), as was found in the body positive condition. In the present

421 study, statements like "I am beautiful" were particularly common in the body positive

422 condition. Such statements could be indicative of participants adopting a broader

423 conceptualisation of beauty to incorporate a variety of appearances and internal attributes

424 when determining beauty in themselves (i.e., 'I am beautiful despite my flaws', 'I am

425 beautiful on the inside', Tylka \& Wood-Barcalow, 2015b), as encouraged by the body

426 positive content they just viewed (i.e., 'every body is beautiful'). Nevertheless, the current

427 coding procedure of the Ten Statements Test limits our ability to clarify what women meant

428 by "I am beautiful" resulting in such statements being coded as appearance-related responses,

429 and thus higher scores of state self-objectification. Qualitative analyses of women's

430 responses to body positive posts would provide a deeper understanding of the impact of this

431 newer media type on women's body image, in particular self-objectification. Moreover,

432 future research is necessary to disentangle the psychological effects of viewing content on

433 social media that reflects aspects of both positive body image and objectification. This

434 inquiry would also help inform and refine existing theories regarding the potential

435 coexistence of these two constructs unique to the body positive environment (Webb, Vinoski,

436 Bonar, Davies, \& Etzel, 2017).

437 


\section{Practical Implications}

In addition to the study's implications for theory and research as discussed above, the

440 current findings have practical implications and reveal a possible constructive avenue for

441 social media use in terms of future prevention and intervention efforts. Unlike traditional

442 media formats whereby users are passive consumers, social media users arguably have

443 agency in terms of what they post and who they follow. The current results suggest that

444 perhaps, as an initial step, simply encouraging women to follow more body positive accounts

445 may help to counterbalance the many idealised messages typical of most women's social

446 media feeds. Our data suggest this is feasible, considering that while only a small percentage

447 of participants reported currently viewing body positive content on their social media, just

448 over half of participants, regardless of exposure condition, said that they were willing to

449 follow body positive accounts in the future. Nevertheless, users should be mindful of the

450 potential for body positive content to increase one's focus on appearance more generally.

\section{$451 \quad$ Limitations and Future Directions}

As with all studies, the present findings should be considered in light of several

453 limitations. Firstly, the study was conducted in a laboratory setting and so, despite using strategies to increase ecological validity, viewing social media posts in an experimental context may not replicate real-word effects. Nevertheless, the positive impact of viewing body positive content was experienced after only three minutes of exposure, whereas, on average, participants reported their typical social media use to be just under two hours a day.

458 Therefore, real life effects of viewing body positive content may be larger than what we 459 found in this study, and future research into the potential longer-term benefits of viewing 460 body positive content would be worthwhile. A second limitation was the lack of pre-exposure 461 measures of state body appreciation and self-objectification, which were purposefully not 462 included to avoid priming and demand characteristics. Moreover, while many efforts were 
made to reduce demand characteristics, participants' responses may still have been

464 influenced by these factors and future research should take this into account. Finally, to enhance ecological validity, stimuli posts were taken directly from Instagram, including both

466 the photograph and caption. However, this approach means it is not possible to differentiate

467 between the impact of the image versus the caption. Similarly, the body positive stimuli were 468 somewhat heterogeneous with three accounts containing images of humans and one account containing images of quotes. Consequently, whilst there appears to be an effect of the body positive stimuli overall, it is difficult to ascertain which types of posts may be driving these effects. Future experimental studies should aim to tease apart these aspects and establish whether both the image and caption are necessary to achieve these effects, and if these effects

473 differ across the various types of body positive posts.

\section{Conclusions}

475 Despite these limitations, the present study demonstrates novel and promising initial 476 findings regarding the effects of viewing 'body positive' content on Instagram on women's 477 mood and body image. Specifically, the findings that exposure to body positive content on 478 Instagram can have a positive impact on women's immediate mood, body satisfaction, and 479 body appreciation significantly extend previous research into 'new' media and body image, 480 as well as contribute to the emerging research into positive body image. Based on the results 481 of the present study, young women who find themselves frequently exposed to thin-ideal 482 content on social media could be encouraged to follow body positive accounts on social 483 media that offer alternative and empowering messages about the body, in order to improve their mood and body image. 
487 Andrew, R., Tiggemann, M., \& Clark, L. (2015). The protective role of body appreciation against media-induced body dissatisfaction. Body Image, 15, 98-104. http://dx.doi.org/10.1016/j.bodyim.2015.07.005

Andrew, R., Tiggemann, M., \& Clark, L. (2016a). Positive body image and young women's health: Implications for sun protection, cancer screening, weight loss and alcohol consumption behaviours. Journal of Health Psychology, 21, 28-39. http://10.1177/1359105314520814

Andrew, R., Tiggemann, M., \& Clark, L. (2016b). Predictors and health-related outcomes of positive body image in adolescent girls: A prospective study. Developmental Psychology, 52, 463.

Aubrey, J. S., Henson, J. R., Hopper, K. M., \& Smith, S. E. (2009). A picture is worth twenty words (about the self): Testing the priming influence of visual sexual objectification on women's self-objectification. Communication Research Reports, 26, 271-284.

Avalos, L., Tylka, T. L., \& Wood-Barcalow, N. (2005). The body appreciation scale: development and psychometric evaluation. Body Image, 2, 285-297. http://dx.doi.org/10.1016/j.bodyim.2005.06.002

Betz, D. E., \& Ramsey, L. R. (2017). Should women be "All About That Bass?": Diverse bodyideal messages and women's body image. Body Image, 22, 18-31. https://doi.org/10.1016/j.bodyim.2017.04.004

Brown, Z., \& Tiggemann, M. (2016). Attractive celebrity and peer images on Instagram: Effect on women's mood and body image. Body Image, 19, 37-43. http://dx.doi.org/10.1016/j.bodyim.2016.08.007 
510 Calogero, R. M. (2013). Objects don't object: Evidence that self-objectification disrupts

$511 \quad$ women's social activism. Psychological Science, 24, 312-318.

512 Calogero, R. M., Herbozo, S., \& Thompson, J. K. (2009). Complimentary weightism: The

513 potential costs of appearance-related commentary for women's self-objectification.

$514 \quad$ Psychology of women quarterly, 33, 120-132.

515 Cohen, R., \& Blaszczynski, A. (2015). Comparative effects of Facebook and conventional

516 media on body image dissatisfaction. Journal of eating disorders, 3, 1-11.

517 http://dx.doi.org/10.1186/s40337-015-0061-3

518 Cohen, R., Newton-John, T., \& Slater, A. (2017). The relationship between Facebook and

519 Instagram appearance-focused activities and body image concerns in young women.

520 Body Image, 23, 183-187. https://doi.org/10.1016/j.bodyim.2017.10.002

521 Cohen, R., Newton-John, T., \& Slater, A. (2018). 'Selfie'-objectification: The role of selfies in self-objectification and disordered eating in young women. Computers in Human Behavior, 79, 68-74. https://doi.org/10.1016/j.chb.2017.10.027

524 Conlin, L., \& Bissell, K. (2014). Beauty ideals in the checkout aisle: Health-related messages in women's fashion and fitness magazines. Journal of Magazine \& New Media Research, 15.

527 Cwynar-Horta, J. (2016). The commodification of the body positive movement on instagram.

528 Stream: Culture/Politics/Technology, 8, 36-56.

529 Fardouly, J., Diedrichs, P. C., Vartanian, L. R., \& Halliwell, E. (2015). Social comparisons on 530 social media: The impact of Facebook on young women's body image concerns and mood. Body Image, 13, 38-45. http://dx.doi.org/10.1016/j.bodyim.2014.12.002 
532 Fredrickson, B. L., Roberts, T.-A., Noll, S. M., Quinn, D. M., \& Twenge, J. M. (1998). That

533

534

535

536

537

538

539

540

541

542

543

544

545

546

547

548

549

550

551

552

553

554 swimsuit becomes you: Sex differences in self-objectification, restrained eating, and math performance. Journal of personality and social psychology, 75, 269.

Fredrickson, B. L., \& Roberts, T. A. (1997). Objectification theory: Toward understanding women's lived experiences and mental health risks. Psychology of women quarterly, 21, 173-206. http://dx.doi.org/10.1111/j.1471-6402.1997.tb00108.x

Grabe, S., Ward, L., \& Hyde, J. S. (2008). The role of the media in body image concerns among women: A meta-analysis of experimental and correlational studies. Psychological Bulletin, 134, 460-476. http://dx.doi.org/10.1037/0033-2909.134.3.460

Groesz, L. M., Levine, M. P., \& Murnen, S. K. (2002). The effect of experimental presentation of thin media images on body satisfaction: A meta-analytic review. International Journal of Eating Disorders, 31, 1-16. http://dx.doi.org/10.1002/eat.10005

Halliwell, E. (2013). The impact of thin idealized media images on body satisfaction: Does body appreciation protect women from negative effects? Body Image, 10, 509-514. http://dx.doi.org/10.1016/j.bodyim.2013.07.004

Halliwell, E. (2015). Future directions for positive body image research. Body Image, 14, 177189. http://dx.doi.org/10.1016/j.bodyim.2015.03.003

Harper, B., \& Tiggemann, M. (2008). The effect of thin ideal media images on women's selfobjectification, mood, and body image. Sex Roles, 58, 649-657. http://dx.doi.org/10.1007/s11199-007-9379-x

Harrison, K., \& Fredrickson, B. L. (2003). Women's sports media, self-objectification, and mental health in black and white adolescent females. Journal of Communication, 53, 216-232. 
Heinberg, L. J., \& Thompson, J. K. (1995). Body image and televised images of thinness and attractiveness: A controlled laboratory investigation. Journal of Social and Clinical Psychology, 14, 325-338.

Holland, G., \& Tiggemann, M. (2016). A systematic review of the impact of the use of social networking sites on body image and disordered eating outcomes. Body Image, 17, 100-110. http://dx.doi.org/10.1016/j.bodyim.2016.02.008

Homan, K. J. (2016). Factor structure and psychometric properties of a state version of the Body Appreciation Scale-2. Body Image, 19, 204-207. http://dx.doi.org/10.1016/j.bodyim.2016.10.004

McLean, S. A., Paxton, S. J., Wertheim, E. H., \& Masters, J. (2015). Photoshopping the selfie: Self photo editing and photo investment are associated with body dissatisfaction in adolescent girls. International Journal of Eating Disorders, 48, 1132-1140. http://dx.doi.org/10.1002/eat.22449

Meier, E. P., \& Gray, J. (2014). Facebook photo activity associated with body image disturbance in adolescent girls. Cyberpsychology, Behavior, and Social Networking, 17, 199-206. http://dx.doi.org/10.1089/cyber.2013.0305

Moradi, B., \& Huang, Y. P. (2008). Objectification theory and psychology of women: A decade of advances and future directions. Psychology of women quarterly, 32, 377398. http://dx.doi.org/10.1111/j.1471-6402.2008.00452.x

Paraskeva, N., Lewis-Smith, H., \& Diedrichs, P. C. (2017). Consumer opinion on social policy approaches to promoting positive body image: Airbrushed media images and disclaimer labels. Journal of Health Psychology, 22, 164-175. http://dx.doi.org/10.1177/1359105315597052 
Pew Research Center. (2018). Social Media Use in 2018. Retrieved from http://www.pewinternet.org/2018/03/01/social-media-use-in-2018/.

Prichard, I., \& Tiggemann, M. (2012). The effect of simultaneous exercise and exposure to thin-ideal music videos on women's state self-objectification, mood and body satisfaction. Sex Roles, 67, 201-210.

Reich, J. W., Zautra, A. J., \& Davis, M. (2003). Dimensions of affect relationships: Models and their integrative implications. Review of General Psychology, 7, 66.

Roberts, T.-A., \& Gettman, J. Y. (2004). Mere exposure: Gender differences in the negative effects of priming a state of self-objectification. Sex Roles, 51, 17-27.

Robinson, L., Prichard, I., Nikolaidis, A., Drummond, C., Drummond, M., \& Tiggemann, M. (2017). Idealised media images: The effect of fitspiration imagery on body satisfaction and exercise behaviour. Body Image, 22, 65-71. https://doi.org/10.1016/j.bodyim.2017.06.001

Slater, A., \& Tiggemann, M. (2015). Media exposure, extracurricular activities, and appearance-related comments as predictors of female adolescents' selfobjectification. Psychology of women quarterly, 39, 375-389.

Stice, E., \& Shaw, H. E. (2002). Role of body dissatisfaction in the onset and maintenance of eating pathology: A synthesis of research findings. Journal of Psychosomatic Research, 53, 985-993.

Swami, V., Weis, L., Barron, D., \& Furnham, A. (2017). Positive body image is positively associated with hedonic (Emotional) and eudaimonic (Psychological and Social) wellbeing in british adults. The Journal of social psychology, 1-12. 
600 Thompson, J., Heinberg, L., Altabe, M., \& Tantleff-Dunn, S. (1999). Exacting beauty: Theory, assessment, and treatment of body image disturbance. Washington, DC, US: American Psychological Association.

Tiggemann, M., \& Boundy, M. (2008). Effect of environment and appearance compliment on college women's self-objectification, mood, body shame, and cognitive performance. Psychology of women quarterly, 32, 399-405.

Tiggemann, M., \& Zaccardo, M. (2015). "Exercise to be fit, not skinny": The effect of fitspiration imagery on women's body image. Body Image, 15, 61-67. http://dx.doi.org/10.1016/j.bodyim.2015.06.003

Tiggemann, M., \& Zaccardo, M. (2018). 'Strong is the new skinny': A content analysis of\# fitspiration images on Instagram. Journal of Health Psychology, 23, 1003-1011.

Tylka, T. L. (2012). Positive psychology perspectives on body image Encyclopedia of body image and human appearance (pp. 657-663): Elsevier.

Tylka, T. L., \& Wood-Barcalow, N. L. (2015a). The Body Appreciation Scale-2: item refinement and psychometric evaluation. Body Image, 12, 53-67. http://dx.doi.org/10.1016/j.bodyim.2014.09.006

Tylka, T. L., \& Wood-Barcalow, N. L. (2015b). What is and what is not positive body image? Conceptual foundations and construct definition. Body Image, 14, 118-129.

Velarde, M. D., Fry, G., \& Tveit, M. (2007). Health effects of viewing landscapes-Landscape types in environmental psychology. Urban Forestry \& Urban Greening, 6, 199-212.

Webb, J. B., Vinoski, E. R., Bonar, A. S., Davies, A. E., \& Etzel, L. (2017). Fat is fashionable and fit: A comparative content analysis of Fatspiration and Health at Every Size ${ }^{\circledR}$ Instagram images. Body Image, 22, 53-64. https://doi.org/10.1016/j.bodyim.2017.05.003 
624 Williamson, G., \& Karazsia, B. T. (2018). The effect of functionality-focused and appearance-

625 focused images of models of mixed body sizes on women's state-oriented body

626 appreciation. Body Image, 24, 95-101.

627

628 
629 Table 1. Means (SD) for state positive mood, negative mood, body satisfaction, body

630 appreciation and self-objectification by exposure condition.

Pre-exposure Post-exposure

\section{Positive Mood}

Body Positive

$68.23(14.16)$

$71.47(16.01)^{\mathrm{a}}$

Thin-ideal

$68.78(17.19)$

$62.30(21.61)^{\mathrm{b}}$

Appearance-neutral

$62.17(19.08)$

$67.09(21.05)^{\mathrm{a}, \mathrm{b}}$

\section{Negative Mood}

Body Positive

$22.87(22.37)$

$20.88(20.61)^{\mathrm{a}}$

Thin-ideal

$22.78(22.02)$

$25.97(23.86)^{\mathrm{a}}$

Appearance-neutral

23.15 (23.08)

$20.18(20.10)^{\mathrm{a}}$

\section{Body Satisfaction}

Body Positive

Thin-ideal

Appearance-neutral
$53.15(20.21)$

$55.02(22.06)$

$52.47(25.38)$ $60.46(21.23)^{\mathrm{a}}$

$47.69(26.03)^{b}$

$54.84(25.40)^{\mathrm{a}, \mathrm{b}}$

\section{Body Appreciation}

Body Positive

Thin-ideal

Appearance-neutral $63.27(19.95)^{\mathrm{a}}$

$52.55(26.30)^{\mathrm{b}}$

$57.10(25.33)^{\mathrm{a}, \mathrm{b}}$

\section{Self-objectification}

Body Positive

Thin-ideal

Appearance-neutral $0.92(0.89)^{\mathrm{a}}$

$0.86(1.06)^{\mathrm{a}}$

$0.37(0.72)^{b}$

$631 \stackrel{*}{p} p<.05,{ }^{* *} p<.001$

632 Note: Means within a column with different superscripts are significantly different at $\mathrm{p}<.05$. 


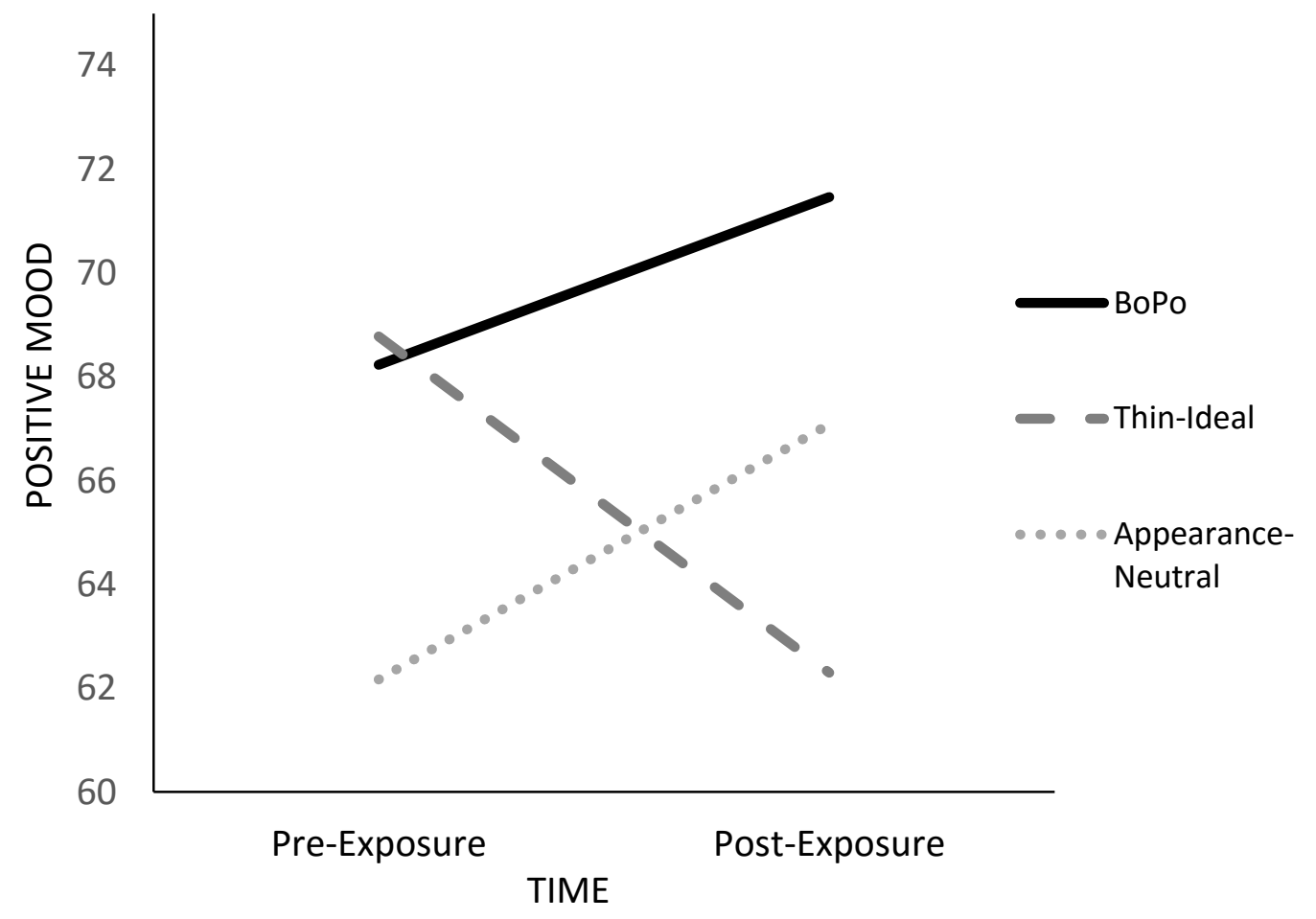

633 Figure 1. Changes in positive mood across time for each exposure condition.

634 


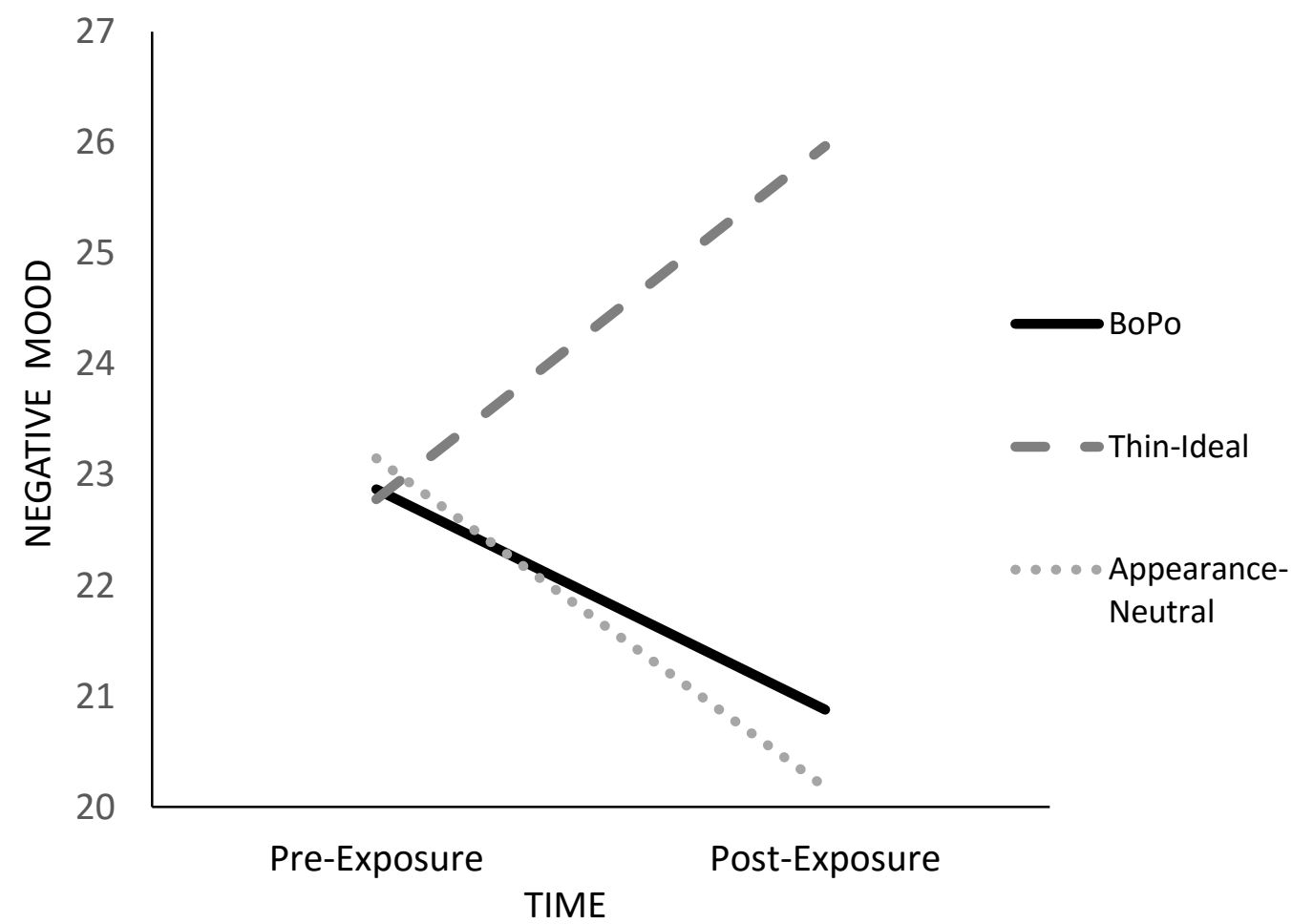

635 Figure 2. Changes in negative mood across time for each exposure condition. 


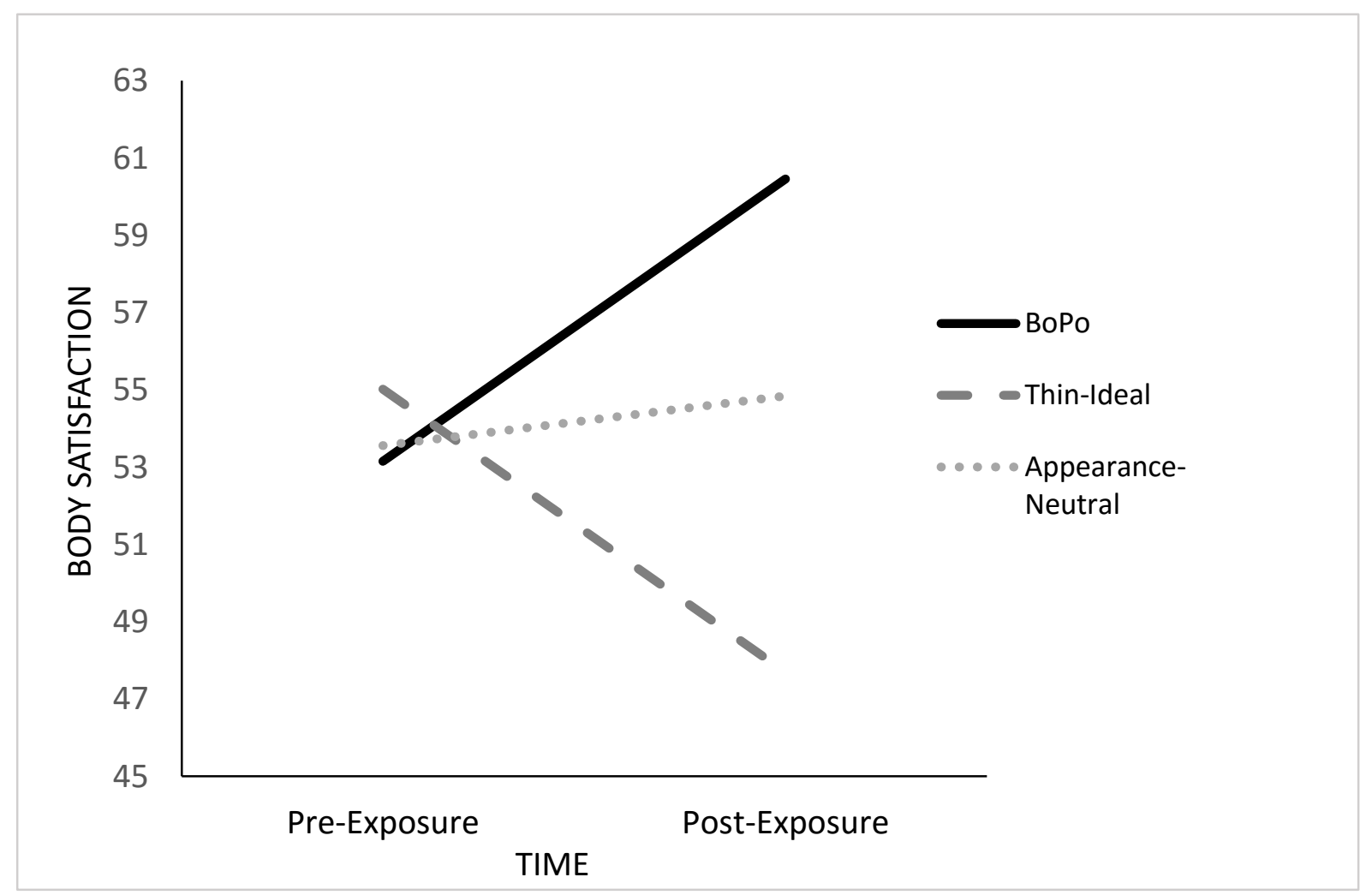

Figure 3. Changes in body satisfaction across time for each exposure condition. 
637

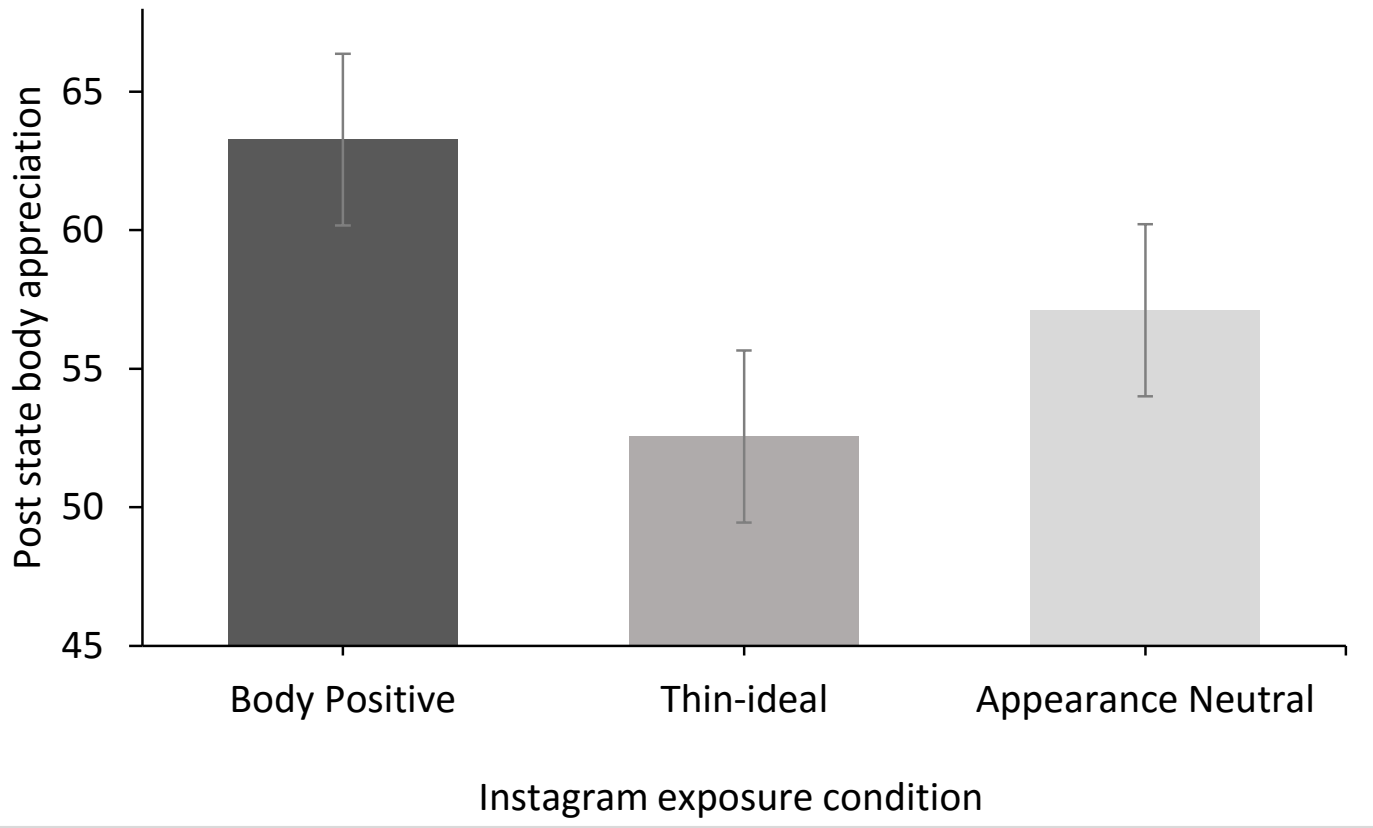

639 Figure 4. Post-exposure scores for state body appreciation for each exposure condition 
640

641

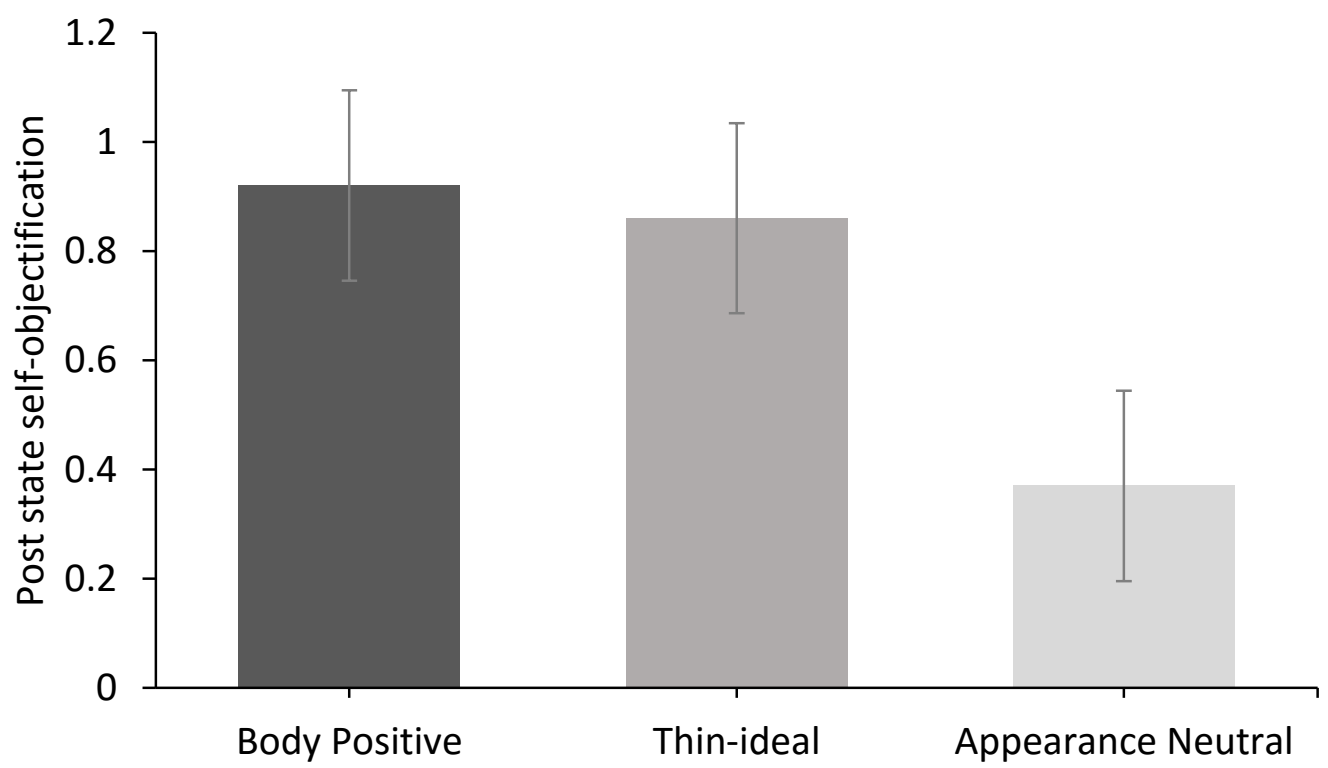

Instagram exposure condition

642 Figure 5. Post-exposure scores for state self-objectification for each exposure condition 